Results The impact of different vaccination strategies on chlamydia population prevalence depends on the characteristics of the vaccine. In the best case scenario, where the vaccine coverage and efficacy is $100 \%$ and duration of protection lifelong, it takes about 7 years to half the prevalence. With an average duration of protection of 10 years, a vaccine coverage or vaccine efficacy of around $70 \%$ or higher per year was needed to half the chlamydia prevalence in 10 years. For high vaccine coverage levels, the impact of vaccinating women alone on population prevalence was greater than vaccinating both men and women. The potential impact of a vaccine on chlamydia population prevalence was sensitive to the duration of protection of the vaccine and the vaccine efficacy.

Conclusion The model suggests that the impact of vaccination strategies on chlamydia prevalence highly depends on characteristics of future vaccines. Current efforts in vaccine development should be accompanied by mathematical models to investigate the optimal strategies.

\section{P1-S4.23 DESCRIBING THE PROGRESSION FROM CHLAMYDIA TRACHOMATIS TO PELVIC INFLAMMATORY DISEASE: SYSTEMATIC REVIEW OF MATHEMATICAL MODELS}

doi:10.1136/sextrans-2011-050108.167

S A Herzog, J C M Heijne, C L Althaus, N Low. University of Bern Bern, Switzerland

Background Chlamydia trachomatis (chlamydia) is an important cause of pelvic inflammatory disease (PID). Preventing PID is a main objective of chlamydia screening. There are many uncertainties about how and when bacteria spread from lower to upper genital tract. The potential impact of screening and treatment, which could interrupt ascending infection, might be affected by the timing of development of PID. Models are often used to investigate the potential impact of screening strategies on PID and should therefore include information about the timing of progression. We conducted a systematic review to determine how the progression from chlamydia to PID is described in mathematical models.

Methods We searched four electronic databases using search terms related to mathematical models and PID from the earliest date to 19 October 2009 without language restrictions. Eligible publications included progression from chlamydia to PID either using a decision tree or a mathematical model. We extracted information about how authors conceptualised the dynamics of chlamydia infection and the development of PID, and assumptions about rates of progression.

Results We identified 41 unique publications about chlamydia infection; 28 of these included PID in a static decision tree. The average percentage of women developing PID in decision analyses was $22.9 \%$ (range $10-35 \%, n=26$ ). For five publications it was not clear how the described model worked. The other eight publications described progression from chlamydia infection to PID dynamically. Of these, two models incorporated PID as a state in a Markov-chain model, four used compartmental models and two used individual-based models. Explicit statements about model structure included the possibility that PID can occur uniformly during a woman's infection, that tubal damage occurs in the second half of the chlamydia infection, and that the model had the ability to vary PID development time. Twenty-eight publications did not mention the stage during a chlamydia infection that progression to PID happens.

Conclusion Most modelling studies do not consider dynamic aspects of $C$ trachomatis transmission and the timing of progression to PID. The mechanisms proposed in studies that made explicit statements could be compared to examine the impact of screening. We suggest that explicit statements about the timing and rates of progression would help improve understanding of the pathogenesis of chlamydial complications and the potential effects of screening.

\section{P1-S4.24 BALANCING THE "SUPPLY AND DEMAND" OF SEX ACTS: IMPLICATIONS FOR MODELLING THE HIV EPIDEMIC AMONG MEN WHO HAVE SEX WITH MEN IN SOUTHERN INDIA}

doi:10.1136/sextrans-2011-050108.168

${ }^{1} \mathrm{~K}$ Mitchell, ${ }^{1} \mathrm{~A}$ Foss, ${ }^{1} \mathrm{H}$ Prudden, ${ }^{2} \mathrm{~J}$ Williams, ${ }^{1} \mathrm{H}$ Johnson, ${ }^{2} \mathrm{M}$ Pickles, ${ }^{2} \mathrm{~A}$ Phillips, ${ }^{3} \mathrm{~B}$ Ramesh, ${ }^{3} \mathrm{R}$ Washington, ${ }^{1} \mathrm{P}$ Vickerman. 'London School of Hygiene and Tropical Medicine, London, UK; ${ }^{2}$ Imperial College London, London, UK; ${ }^{3}$ Karnataka Health Promotion Trust, Bangalore, India

Background In India, men who have sex with men (MSM) have distinct identities related to the role taken in anal sex (Panthi/ Bisexual (PB): mostly insertive, Kothi/Hijra $(\mathrm{KH})$ : mostly receptive, Double Deckers: both). Wide discrepancies are found between the supply and demand for sex acts estimated for each group using data on reported frequency of anal sex, role taken and estimated group population sizes.

Methods Two methods for balancing the number and type of sex acts between different groups were compared. They were used in a deterministic HIV transmission model to estimate mixing patterns and HIV prevalence over the first 20 years of the epidemic (including reported condom use trends) and a subsequent 10-year intervention $(10 \%$ absolute increase in condom use). Data collected from Bangalore for the evaluation of Avahan (the India AIDS initiative) on the mean reported frequency of sex acts per individual, role taken in anal sex and population sizes for each group were used to construct a mixing matrix. In method $\mathrm{A}$, the $\mathrm{PB}$ group size was set to balance the total number of insertive and receptive acts, and receptive acts for each group were distributed among the three groups in proportion to the number of insertive acts offered. In method $\mathrm{B}$, the proportion of receptive acts $\mathrm{KH}$ had with other $\mathrm{KH}$ was an additional input parameter, with remaining receptive acts distributed as in method A. The number and type of contacts for all groups were adjusted to achieve balancing. The model was run using 300000 randomly sampled parameter sets drawn from the data and multiple fits were found to group-specific HIV prevalence data.

Results Model fits for method B had more assortative (like-with-like) mixing than method A, particularly for $\mathrm{PB}$ (median number of acts $\mathrm{PB}$ have with other $\mathrm{PB}$ : $48.5 \%$ (IOR 33.3-63.3\%) in A, 63.3\% (IOR $47.3-74.1 \%$ ) in $\mathrm{B}$ ), related to larger $\mathrm{PB}$ group sizes and $\mathrm{PB}$ taking the insertive role less often in B. Despite these differences, the fitted epidemic curves were very similar for all three groups across the two methods (Absrtact P1-S4.24 figure 1), as was the predicted

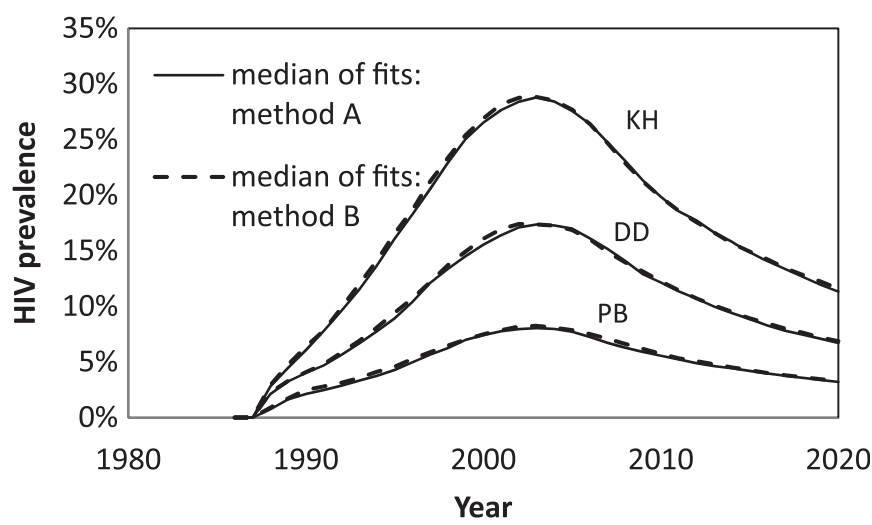

Abstract P1-S4.24 Figure 1 MSM. 